\title{
Biomechanics-based active control of bedding support properties and its influence on sleep
}

\author{
Van Deun D..$^{\text {a, }}$, Verhaert V. ${ }^{\text {a }}$, Willemen T. ${ }^{\mathrm{a}}$, Wuyts J. ${ }^{\mathrm{b}}$, Verbraecken J. ${ }^{\mathrm{c}}$, Exadaktylos V. ${ }^{\mathrm{d}}$, Haex B. ${ }^{\mathrm{a}}$ and \\ Vander Sloten J. ${ }^{\mathrm{a}}$ \\ ${ }^{a}$ Biomechanics section, Katholieke Universiteit Leuven, Celestijnenlaan 300c, bus 2419, 3001 Leuven, Belgium \\ ${ }^{\mathrm{b}}$ Biological Psychology, Vrije Universiteit Brussel, Pleinlaan 2, 1050 Brussels, Belgium, \\ ${ }^{c}$ Multidisciplinary Sleep Disorders Center, Antwerp University Hospital and University of Antwerp, Wilrijkstraat \\ 10, 2650 Edegem, Belgium, \\ ${ }^{d}$ M3-BIORES, Katholieke Universiteit Leuven, Kasteelpark Arenberg 30, 3001 Leuven, Belgium
}

\begin{abstract}
Proper body support plays an import role in the recuperation of our body during sleep. Therefore, this study uses an automatically adapting bedding system that optimises spinal alignment throughout the night by altering the stiffness of eight comfort zones. The aim is to investigate the influence of such a dynamic sleep environment on objective and subjective sleep parameters. The bedding system contains 165 sensors that measure mattress indentation. It also includes eight actuators that control the comfort zones. Based on the measured mattress indentation, body movements and posture changes are detected. Control of spinal alignment is established by fitting personalized human models in the measured indentation. A total of 11 normal sleepers participated in this study. Sleep experiments were performed in a sleep laboratory where subjects slept three nights: a first night for adaptation, a reference night and an active support night (in counterbalanced order). Polysomnographic measurements were recorded during the nights, combined with questionnaires aiming at assessing subjective information. Subjective information on sleep quality, daytime quality and perceived number of awakenings shows significant improvements during the active support (ACS) night. Objective results showed a trend towards increased slow wave sleep. On the other hand, it was noticed that \% N1-sleep was significantly increased during ACS night, while \% N2-sleep was significantly decreased. No prolonged N1 periods were found during or immediately after steering.
\end{abstract}

Keywords: Smart bedding, spine support, sleep quality, spinal alignment, mattress control

\section{Introduction}

Although our living environment is continuously evolving towards high-tech systems to provide optimal comfort, only little research focuses on the sleep environment. On the other hand, both sleep physiology and ergonomics are continuously growing research fields. Yet, at present there is a lack of knowledge about the impact of the sleep surface on general sleep quality. As a consequence, the question remains whether applying ergonomic design principles affects actual sleep (i.e. sleep quality, sleep architecture, movements, etc).
According to Webster's dictionary, the primary definition of comfort is "the provision of support and assistance". Applied to the design of bedding systems, improving comfort involves providing optimal body support. Optimal support refers to aligning the spine towards its reference shape, which is comparable to the shape while standing but with a slightly flattened lumbar lordosis due to the changed working axis of gravity [6]. However, bed properties that provide optimal support are posture- and person-dependent. For instance, in most persons coronal and sagittal contours differ, which results in a changed spinal alignment after changing sleep posture $[8,20]$. To

Corresponding author. E-mail: dorien.vandeun@mech.kuleuven.be 
account for these variable conditions, a new approach in the development of state-of-the-art bedding systems consists of monitoring sleep posture changes based on mattress indentation data. In addition, the combination of mattress indentation with personalised human models allows estimating spinal alignment during sleep in an unobtrusive way [21].

This study makes use of a bedding system that autonomously alters its stiffness distribution according to the estimated spinal alignment. The aim of this study is to investigate the effect of such a "smart" bedding system on sleep in both an objective and a subjective way through polysomnography and questionnaires respectively. Also movement and posture information is gathered using the specified bedding system.

\section{Methodology}

\subsection{Participants}

A total of 11 volunteers (6 males, 5 females) between the age of 20 and 28 years (mean age $21.2 \pm$ 3.2 y) participated in this study. Subjects were recruited through advertisement. All volunteers were healthy sleepers. Exclusion criteria consisted of medical problems that can interfere with normal sleep, e.g. regular smoking, abuse of alcohol, any form of back pain and the intake of sleep medication or antidepressants. Adherence to these standards was ensured using the Pittsburgh Sleep Quality Index [4], the Insomnia Interview Schedule [15] and a general intake interview. All participants signed informed consent forms. The study was approved by the Ethics Committee of the Vrije Universiteit Brussel.

\subsection{Experimental design}

Each subject slept three nights in a sleep laboratory: a first night, considered as habituation night, a reference night (REF) - in which the sleep system was set in its standard configuration - and an "active support" night (ACS) where the sleep system was working automatically towards optimal spine support. Counterbalancing was applied to avoid carry-over effects between conditions. Participants slept one night at home between each night in the laboratory and were blinded from experimental conditions. The experimental nights consisted of approximately eight hours time in bed. The first night in the sleep labora- tory was not used for further analysis of the results [1].

\subsection{Procedure}

Two weeks prior to the experiment and during the experimental week, participants had to fill out sleep diaries to check for any abnormalities in their sleepwake patterns. Two dimensional body contours in both the sagittal and the coronal plane were automatically registered by means of silhouette extraction using an optical measurement system (Ikélo, Custom8, Leuven, Belgium). These body contours provided the necessary anthropometric input to generate a personalised human model (Figure 1) [21].

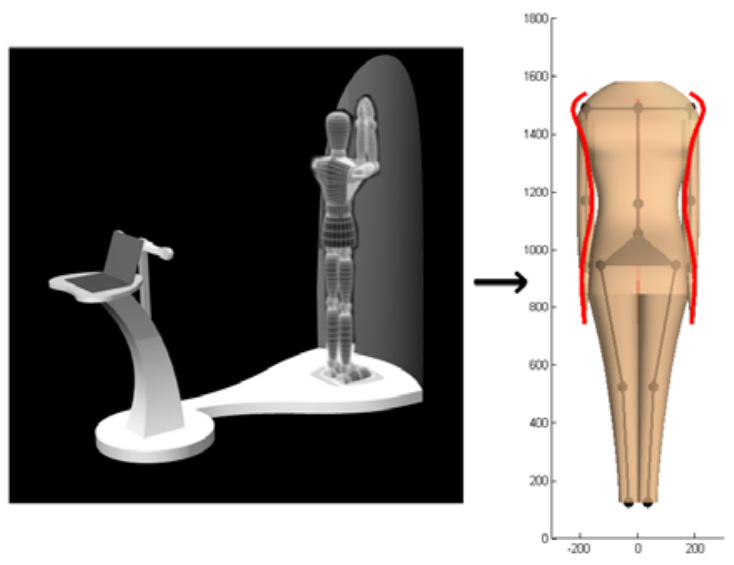

Figure 1

Silhouette extraction and personalized human model generated from coronal and sagittal body contours using the Ikélo system. Adapted from Verhaert et al. 2011 [21]

Subjects filled out questionnaires at various moments throughout the course of the experiments. At $19 \mathrm{~h} 00$ and upon retiring to bed (between 22h30 and 23h30) they completed a Karolinska Sleepiness Scale (KSS) [2], a Profile of Mood State (POMS) [5], a Stress/Arousal Adjective Checklist (SA-CL) [12] and an Activation/Deactivation Adjective Checklist (ADACL) [14]. In the morning following the test night (around 07h20) the subjects repeated the above questionnaires. In addition, they completed a sleep diary and were questioned regarding general sleep quality (SQ) and bed comfort. Finally, alertness and sleepiness during the day was questioned in the evening following the test night. The questionnaires were rescaled in such a way that high values implicate positive results (less fatigued, more rested, better 
sleep quality,...). This means that when differences between the evening before and the morning following the test night are reported (see Table 2), a negative difference value corresponds to a restorative effect of the night's sleep.

\subsection{Measurements}

Polysomnographic measurements (PSG) were performed during the nights (including EEG, EOG, skin temperature, ECG and EMG measurements). Furthermore, video recording as well as chest orientation were measured. All channels were measured at 200 $\mathrm{Hz}$ using a customised Medatec DREAM system (Medatec, Brussels, Belgium).

Specifically designed sleep systems were used, equipped with sensors and actuators. A total of 165 linear potentiometers, arranged in a staggered twodimensional grid of 11 by 30 , measured the perpendicular indentation of the mattress core (DynaSleep, Custom8, Leuven, Belgium). Indentation was continuously measured and recorded throughout the nights at a sampling rate of $1 \mathrm{~Hz}$. The main sleep postures (left/right lateral, prone, supine) were estimated from the indentation data using a Support Vector Machine (SVM) classifier, that used five features according to the classification scheme proposed by Verhaert et al. [22]. This algorithm allows posture detection with an overall accuracy of $92 \%$. Based on the detected posture, spinal alignment was estimated by fitting the model in the measured indentation [21].

During the "active support" (ACS) night actuators in the bedding system enabled adaptation of the stiffness in eight zones of the mattress in order to continuously optimise the spine shape. Figure 2 presents an overview of the control that is implemented in the bed systems. The general input of the control algorithm is provided by the mattress indentation measurements, from which posture and spine shape can be calculated using the personalized human models (more detailed information on this procedure can be found in [23]). Based on a comparison of the modelled spine shape with the desired (reference) shape a new bed configuration is proposed (i.e. new target values are forwarded to the actuators). Steps II to IV are repeated until the modelled shape approximates the reference shape close enough.

The active steering started 30 minutes after lights out to avoid a prolonged sleep onset due to the steering.

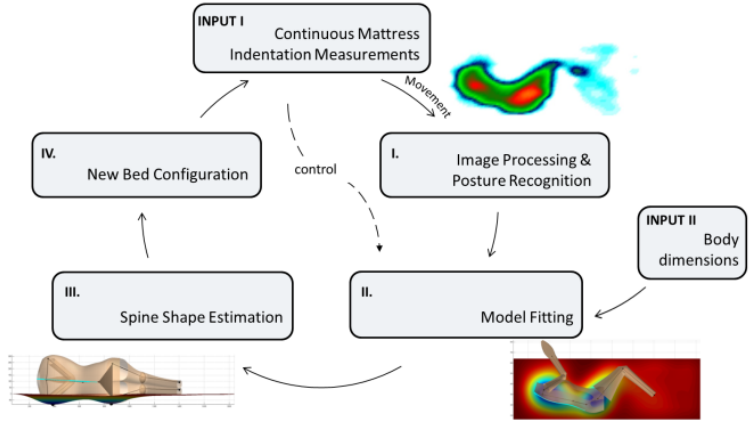

Figure 2

Schematic overview of the working principle of the implemented control loop for the alteration of bed properties during sleep. Based on mattress indentation measurements, movements are detected. Postures are estimated when a stable position is achieved for at least 10 seconds. Afterwards, a personalised human model is fitted in the indentation surface in order to estimate spine shape. Based on this estimated shape, the decision is made whether or not to adjust the bedding system.

\subsection{Data analysis}

From the recorded mattress indentation a movement signal is derived, which is defined by the summation of the rectified time derivative of each potentiometer output. The signal is then scaled according to the Body Mass Index (BMI) of the sleeper (Eq. (1)), and used to calculate different movement parameters, such as the number of body movements. Small, medium and large movements were determined by applying a threshold of respectively 2, 10 and 40 times the mean of the signal.

$$
\text { mowenent signal }=\frac{\sum_{\mathrm{i}-1}^{4 c z}\left|\frac{\text { d(natantimeter mathat) }}{d t}\right|}{D M I} \operatorname{cst}
$$

PSG output was scored according to Rechtschaffen $\&$ Kales [16], adopted to the revision of the American Academy of Sleep Medicine [11]. Scoring was performed by a trained professional and the resulting sleep hypnogram was used to calculate objective sleep parameters. Normality was checked with a Lilliefors normality test, while a Bartlett's t-test provided information on whether the variances of both groups were equal. In case of a normal distribution with equal variances, two-sided student's t-tests were used to compare sleep parameters between the active support night (ACS) and the reference night (REF) 
within subjects. A Wilcoxon signed-rank test was used as a non-parametric alternative. Differences of $p$ $<0.05$ were considered significant for all statistical analyses.

\section{Results}

\subsection{Subjective assessment}

Sleep quality, as questioned on a scale of zero (extremely bad) to ten (extremely good), showed a significant increase $(p=0.029)$ in the ACS night (Table 1). Other subjective results derived from the analysis of the sleep diaries showed that subjects perceived significantly less awakenings $(p=0.041)$ during the ACS night and pointed out that night-time awakenings were shorter $(p=0.045)$. Table 2 lists the main results of the questionnaire data. Although all results were in favour of the ACS condition, statistical sig-

Table 1

\begin{tabular}{c|ccc}
\multicolumn{4}{c}{ Subjective results from sleep diary } \\
& $\begin{array}{c}\text { Mean REF } \\
\text { night }\end{array}$ & $\begin{array}{c}\text { Mean ACS } \\
\text { night }\end{array}$ & p-value \\
\hline \hline $\begin{array}{c}\text { Subjective SOL [15 } \\
\text { min] }\end{array}$ & $0.80 \pm 0.79$ & $1.10 \pm 0.88$ & $0.430^{\mathrm{w}}$ \\
$\begin{array}{c}\text { Subjective number } \\
\text { of Awakenings [-] }\end{array}$ & $1.70 \pm 0.95$ & $0.80 \pm 0.79$ & $0.041^{\mathrm{t}}$ \\
$\begin{array}{c}\text { Subjective mean } \\
\text { awakening length } \\
\text { [min] }\end{array}$ & $1.25 \pm 0.63$ & $0.90 \pm 0.88$ & $0.045^{\mathrm{t}}$ \\
$\begin{array}{c}\text { Subjective Sleep } \\
\text { quality [0-10] }\end{array}$ & $6.60 \pm 1.63$ & $7.5 \pm 1.32$ & \\
\hline
\end{tabular}

${ }^{\mathrm{t}}$ Two-sided paired t-test; ${ }^{\mathrm{w}}$ Wilcoxon signed-rank test

nificance was only reached for daytime quality following the test night.

\subsection{Objective assessment: sleep architecture}

Table 3 presents the sleep parameters derived from the hypnogram. The time spent in N1-sleep is signify
Table 2

Subjective sleep parameters derived from questionnaire data (morning after, evening before and/or evening

\begin{tabular}{|c|c|c|c|}
\hline & $\begin{array}{l}\text { Mean } \\
\text { REF night }\end{array}$ & $\begin{array}{c}\text { Mean } \\
\text { ACS night }\end{array}$ & p- value \\
\hline $\begin{array}{c}\text { Daytime quality score } \\
\text { EA [/ 30] }\end{array}$ & $22.35 \pm 4.86$ & $24.36 \pm 4.4$ & $0.039^{w}$ \\
\hline $\begin{array}{l}\text { POMS fatigue difference } \\
\text { EB-M }[-5-5]\end{array}$ & $0.15 \pm 0.67$ & $0.00 \pm 0.76$ & $0.623^{\mathrm{w}}$ \\
\hline $\begin{array}{c}\text { AD-ACL Arousal } \\
\text { difference EB-M [-5 - 5] }\end{array}$ & $-0.34 \pm 1.04$ & $-0.53 \pm 1.08$ & $0.554^{\mathrm{t}}$ \\
\hline $\begin{array}{c}\text { KSS Sleepiness } \\
\text { difference EB-M [-5 - 5] }\end{array}$ & $-0.72 \pm 1.07$ & $-0.95 \pm 1.01$ & $0.513^{\mathrm{w}}$ \\
\hline $\begin{array}{c}\text { KSS Sleepiness } \\
\text { difference EB-EA[-5 - 5] }\end{array}$ & $-0.95 \pm 1.09$ & $-1.38 \pm 1.03$ & $0.137^{\mathrm{t}}$ \\
\hline $\begin{array}{c}\text { Bedding system Quality } \\
\text { M }[/ 10]\end{array}$ & $4.53 \pm 1.65$ & $4.95 \pm 1.68$ & $0.310^{\mathrm{w}}$ \\
\hline $\begin{array}{l}\text { Feeling rested score } \mathrm{M}[0 \\
-10]\end{array}$ & $6.71 \pm 1.87$ & $7.24 \pm 1.92$ & $0.248^{\mathrm{t}}$ \\
\hline $\begin{array}{c}\text { Back pain score M [1 (a } \\
\text { lot) }-4 \text { (none) }]\end{array}$ & $1.86 \pm 1.56$ & $2.39 \pm 1.40$ & $0.180^{\mathrm{w}}$ \\
\hline
\end{tabular}

cantly $(\mathrm{p}=0.027)$ increased with approximately $2 \%$ in the ACS night. Percentage N2, on the other hand, was significantly decreased $(p=0.014))$, while percentage slow wave sleep (SWS) tended to increase in the ACS night $(p=0.096)$. No other effects were present in terms of sleep macrostructure.

\subsection{Movement and sleep postures}

The number of body movements (small, medium or large BMs, Table 3) was not statistically different between conditions. Furthermore no significant changes were present in the amount of time that was spent in each sleep posture (Table 4), nor in the number of posture changes (Table 3 ). 
Table 3

Objective parameters derived from PSG and movement

\begin{tabular}{|c|c|c|c|}
\hline & $\begin{array}{c}\text { Mean } \\
\text { MEF night } \\
\text { Ren }\end{array}$ & $\begin{array}{c}\text { Mean } \\
\text { ACS night }\end{array}$ & $\mathrm{p}$-value \\
\hline SOL [min] & $11.78 \pm 4.78$ & $13.22 \pm 12.67$ & $0.98^{\mathrm{w}}$ \\
\hline N2-latency[min] & $14.17 \pm 5.23$ & $\begin{array}{c}16.302 \pm \\
5.232\end{array}$ & $0.75^{\mathrm{t}}$ \\
\hline LPS [min] & $13.67 \pm 5.59$ & $\begin{array}{c}17.145 \pm \\
5.588\end{array}$ & $0.33^{\mathrm{w}}$ \\
\hline LDS [min] & $26.12 \pm 7.60$ & $29.49 \pm 7.60$ & $0.31^{t}$ \\
\hline $\begin{array}{l}\text { Total Sleep } \\
\text { Time [min] }\end{array}$ & $423.5 \pm 30.90$ & $423.55 \pm 30.9$ & $0.68^{\mathrm{w}}$ \\
\hline $\begin{array}{l}\text { Intrasleep Wake } \\
\text { time [min] }\end{array}$ & $37.37 \pm 30.16$ & $34.29 \pm 30.16$ & $0.44^{t}$ \\
\hline AWAKE [\%] & $8.07 \pm 5.22$ & $4.44 \pm 5.99$ & $0.56^{\mathrm{w}}$ \\
\hline N1 [\%] & $7.91 \pm 5.12$ & $9.86 \pm 5.77$ & $0.027^{\mathrm{w}}$ \\
\hline $\mathrm{N} 2[\%]$ & $51.75 \pm 8.27$ & $44.85 \pm 5.970$ & $0.014^{t}$ \\
\hline SWS [\%] & $24.50 \pm 5.89$ & $29.89 \pm 6.19$ & $0.096^{\mathrm{w}}$ \\
\hline NREM [\%] & $76.25 \pm 6.90$ & $74.73 \pm 6.34$ & $0.45 \mathrm{t}^{\mathrm{t}}$ \\
\hline REM [\%] & $15.78 \pm 8.28$ & $15.29 \pm 7.20$ & $0.77^{\mathrm{t}}$ \\
\hline BM Small [-] & $46.10 \pm 32.10$ & $55.00 \pm 23.82$ & $0.16^{\mathrm{t}}$ \\
\hline BM Medium [-] & $34.3 \pm 27.17$ & $32 \pm 19.56$ & $0.61^{t}$ \\
\hline BM large [-] & $30.00 \pm 9.07$ & $27.5 \pm 5.60$ & $0.20^{t}$ \\
\hline $\begin{array}{l}\text { Nr of posture } \\
\text { changes }\end{array}$ & $13.00 \pm 7.47$ & $12.1 \pm 4.07$ & $0.57^{\mathrm{w}}$ \\
\hline
\end{tabular}

${ }^{\mathrm{t}}$ Two-sided paired t-test; ${ }^{\mathrm{w}}$ Wilcoxon signed-rank test

SOL: Sleep onset latency; LPS: latency to persistent sleep; LDS: latency to deep sleep (SWS); TST: total sleep time; TIB: time in bed; W: wake; N1: stage N1 sleep; N2: stage N2 sleep; SWS: slow wave sleep ; NREM: non rapid eye movement; REM: rapid eye movement; BM: body movement.

\section{Discussion}

This study aimed at exploring the influence of an active bedding system - designed to continuously optimise spinal alignment - on objective and subjective sleep parameters. Overnight experiments in a dedicated sleep laboratory were performed in order to look at sleep macrostructure, body movements and subjective parameters.

Subjective results showed an improvement in the feeling that people have about their sleep in the morning following a night on an actively steered bedding system. Moreover, daytime quality after sleeping on the active system increased significantly.

Movement information showed no significant differences between conditions. No within-subject changes were found in the amount of time spent in
Table 4

\begin{tabular}{c|cccc}
\multicolumn{5}{c}{ Adopted sleep postures in REF and ACS night } \\
& Supine & $\begin{array}{c}\text { Left } \\
\text { lateral }\end{array}$ & $\begin{array}{c}\text { Right } \\
\text { lateral }\end{array}$ & prone \\
\hline \hline \multirow{2}{*}{ REF night [\%] } & $28.20 \pm$ & $26.82 \pm$ & $24.68 \pm$ & $6.30 \pm$ \\
& 19.03 & 15.87 & 19.51 & 11.21 \\
& & & & \\
ACS night [\%] & $27.19 \pm$ & $25.00 \pm$ & 24.80 & $5.91 \pm$ \\
& 14.93 & 15.23 & \pm 12.95 & 12.77
\end{tabular}

each sleep posture. The large standard deviations for the occurrence of sleep postures (Table 4) reflect large interpersonal differences in habitual sleep postures. This confirms the need for an individualised (and thus actively steered) approach of bedding systems, as presented in this study.

The sleep hypnogram revealed a trend towards more SWS during the ACS night. Since SWS, or deep sleep, is important for physical recuperation [18] these results might indicate a positive restorative effect of the active support. Some studies investigating the effect of bedding systems on sleep failed to find any differences in objective PSG parameters [17]. Others found differences in \% SWS during sleep on different bedding systems, but did not incorporate quantified validation of spinal alignment [19, 13]. Tsai and Liu [19] reported differences in SWS in subjects with mild sleep-related respiratory disturbances sleeping on bedding systems that were selected by means of manual muscle testing. Lee and Park [13] found a significant increase in SWS after replacing the mattress with "one that can maintain spinal curvature in a manner more similar to that found while standing". However, they did not provide a quantification of spinal curvature, nor specify how they assessed it.

Furthermore, an increase in percentage N1 sleep was seen during the ACS night. It is known that during and immediately after a posture change subjects are in an elevated state of arousal [9]. Since the current steering control awaited only 10 seconds after the end of each posture change, it is plausible that the subjects were still in an elevated state of arousal when the actuators were working and became aware of the changing bed properties, causing the arousal to prolong. However, no differences in wakefulness, nor in duration of N1 after steering were noted.

Nevertheless, further analysis in terms of microstructural sleep parameters - looking into the energy contained in brain waves - during and immediately after steering should be performed in order to check whether steering has an effect on sleep microstructure. If an increase in alpha-activity is apparent in these 
periods, a possible solution might be to wait until the sleeper has (re)entered a deeper sleep stage before changing bed properties.

One can argue whether objective or subjective results are in favour to draw any conclusions on sleep evaluation. Subjective sleep quality depends a lot on the perceived time awake after sleep onset [5], which is also reflected in the results of this study (Table 1). People thus perceive a good sleep quality when sleep continuity is assured [2]. However, this is not always reflected by objective sleep parameters.

Finally, the following recommendations for future research are proposed. First, it should be stressed that this study only investigated a one-night effect. Long term studies are needed to look at long-term effects of optimized support by means of active bedding systems. Second, other factors than spinal alignment have been proposed in relation to sleep comfort, for instance pressure distribution [3, 13]. Therefore, ongoing research is exploring how changing the sleep system's stiffness distribution alters pressure distribution. Finally, specific patient populations should be studied (e.g. low-back pain sufferers), since it has been shown that improved body support is especially beneficial for people with chronic low-back pain or non-specific sleep complaints [7].

\section{Conclusion}

This study aimed at identifying the effects of an actively steered bedding system on objective and subjective sleep parameters. Results showed a positive effect on subjectively perceived sleep and a trend towards more slow wave sleep. On the other hand, a small, yet significant increase in N1 sleep was noted. Since no prolonged N1 periods were found during or immediately after steering, further analysis in terms of micro-structural sleep parameters in these periods is needed to investigate whether subjects became aware of the changing sleep environment during the night.

\section{Acknowledgements}

This research was carried out thanks to the financial support of the Institute for the Promotion of Innovation through Science and Technology in Flanders (IWT).

\section{References}

[1] Agnew, H.W., Webb, W.B., Wiliams, R.L. (1966). The first night effect: an EEG study of sleep, Psychophysiology 2, 263 $-266$

[2] Äkerstedt T., Huma K., Minors D., Waterhouse J. (1994), The meaning of good sleep: a longitudonal study of polysomnography and subjective sleep quality, Journal of Sleep Research $3-3,152-158$

[3] Buckle P. And Fernandes A. (1998), Mattress evaluation assessment of contact pressure, comfort and discomfort, Applied Ergonomics 29, 35 - 39

[4] Buysse, D.J., Reynolds, C.F., Monk, T.H., Berman, S.R., Kupfer, D.F. (1989), The Pittsburgh Sleep Quality Index: a new instrument for psychiatric practice and research, Psychiatry Res. 28 (2), $193-213$

[5] Cluydts R. (1979). Gemoedstoestanden en slaap.Proefschrift, Vrije Universiteit Brussel, Brussel

[6] Dolan, P., Adams, M.A., Hutton, W.C. (1988), Commonly adopted postures and their effect on the lumbar spine, Spine, 13, 197-201.

[7] Enck, P., Walten, T., Traue, H.C. (1999). Associations between back pain, quality of sleep and quality of mattresses. Double-blind pilot study with hotel guests, Schmerz 13, 205 207

[8] Haex, B. (2004). Back and Bed: Ergonomic Aspects of Sleeping, Boca Raton: CRC Press

[9] Hobson, J. Allan (2005). Sleep is of the brain, by the brain and for the brain, Nature 437, 1254 - 1256

[10]Lee, H.,, Park, S. (2006). Quantitative effects of mattress types (comfortable vs. uncomfortable) on sleep quality through polysomnography and skin temperature, International Journal of Industrial Ergonomics, 36, 943-949

[11]Iber, C., Ancoli-Israel, S., Chesson, AL., Quan, SF. (2007). The AASM Manual for the Scoring of Sleep and Associated Events. Rules, Terminology and Technical Specifications, 1st ed: Westchester, Illinois: American Academy of Sleep Medicine.

[12]King, M.G., Burrows, G.D., and Stanley, G.V. (1983). Measurement of stress and arousal: Validation of the stress/arousal adjective checklist. Br J Psychol, 74, 473 - 479

[13] Lee, H., Park, S. (2006). Quantitative effects of mattress types (comfortable vs. Uncomfortable) on sleep quality through polysomnography and skin temperature, International Journal of Industrial Ergonomics 36, $943 \quad-\quad 949$, doi:10.1016/j.ergon.2006.07.007

[14] Mackay, C., Cox, T., Burrows, G., and Lazzerini, T. (1978). An inventory for the measurement of self-reported stress and arousal. Br J Soc Clin Psychol, 17 (3), 283 - 284

[15]Morin, C.M. (1993), Insomnia: Psychological assessment and management. New York, NY: The Guilford Press.

[16]Rechtschaffen, A., Kales, A. (1968). A manual of standardized terminology, techniques and scoring system of sleep stages in human subjects. Los Angeles: Brain Information Service/Brain Research Institute, University of California.

[17] Scharf, M. B., Stover, R., McDannold, M., Kaye, H., Berkowitz, D.V. (1997) Comparative Effects of Sleep on a Standard Mattress to an Experimental Foam Surface on Sleep Architecture and CAP Rates1197-1200, Sleep 20, 1197-1200

[18] Shapiro, CM., Bortz, R., Mitchell, D., Bartel, P., and Jooste, P. (1981). Slow-wave sleep: a recovery period after exercise, Science 214 (4526), $1253-1254$, doi: $10.1126 /$ science. 7302594 
[19]Tsai, L.L. and Liu H.M. 2008). Effects of bedding systems selected by manual muscle testing on sleep and sleep-related respiratory disturbances, Applied Ergonomics 39, 261 - 270

[20] Verhaert, V., Haex, B., De Wilde, T., Berkmans, D., Verbraecken, J., de Valck, E., Vander Sloten, J. (2011a). Ergonomics in bed design: the effect of spinal alignment on sleep parameters, Ergonomics 54, $169-178$

[21]Verhaert, V., et al. (2011b). The use of a generic human mode to personalize bed design. Accepted for publication in Proceedings of 1st International Symposium on Digital Human Modeling, Lyon.

[22] Verhaert, V., Haex, B., De Wilde, T., Berckmans, D., Vandekerckhove, M., Verbraecken, J., and Vander Sloten, J. (2011c). Unobtrusive assessment of motor pattern during sleep based on mattress indentation measurements. IEEE T. Inf. Technol. B., in press, doi: 10.1109/TITB.2011.2131670.

[23]Verhaert, V., Van Deun, D., Verbraecken, J., Michiels, N., Vandekerckhove, M., Exadaktylos, V., Haex, B. and Vander Sloten, J. (under review), Smart control of spinal alignment by adapating mechanical bed properties during sleep, Journal of Ambient Intelligence and Smart Environments 